\title{
Alternative Fakten und Narrative Ethik
}

\section{Rouven Porz}

PD Dr. phil., dipl. biol., Leiter der Fachstelle für klinische Ethik der Insel Gruppe AG (Bern), Gastwissenschaftler der VU Amsterdam, Generalsekretär der European Association of Centres of Medical Ethics (EACME) und Mitglied der Redaktion Ethik der SÄZ

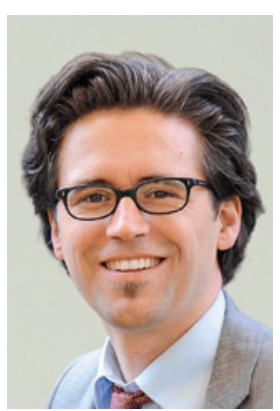

Ich glaube, dass Donald Trump den Ethikunterricht verändert hat. Also zumindest meinen Unterricht, den ich als Ethikdozent zu gestalten suche. Bisher war mein Ethikunterricht immer darauf aufgebaut, dass ich die Ethik als wissenschaftliche Disziplin einführe, als Unterscheidung zwischen Fakten und Werten, als Reflexion von Pro- und Kontra-Argumenten, als rationale Auslegeordnung, die sich mit Prinzipien, Folgerichtigkeit, Konsequenzen und Transparenz beschäftigt; dies immer im Ideal der Suche nach einem möglichst guten Zusammenleben zwischen Menschen.

Die Ergebnisse der US-Wahlen haben jetzt diesem Verständnis irgendwie auf den ersten Blick etwas den Rang abgelaufen, weil man - so ehrlich muss man sein, und einige Studenten haben das auch schon geäussert sehr, sehr mächtig in dieser westlichen Welt werden kann ohne diese Art der Ethik, also anscheinend ohne kohärente Argumente und konsequente Rationalität; einfach in dem man Geschichten erzählt, die die Leute anscheinend überzeugen. Klar, so war die Politik vielleicht schon immer, aber mit den Wahlen in den USA wurde es jetzt für uns alle irgendwie offensichtlicher: "Alternative Fakten" ist das neue Stichwort. Also man erzählt einfach irgendetwas und behauptet dann fest, dass es so gewesen sei.

Gut nur, dass uns auch hier die Disziplin der Ethik helfen kann, um alternative Fakten als solche zu entlarven. Man muss nur etwas tiefer in die Kiste des ethischen Methodenrepertoires greifen. Helfen kann uns die sogenannte Narrative Ethik. Unter Narrativer Ethik kann ein Zugang verstanden werden, der den Wert- und Normvorstellungen von Geschichten besondere ethische Beachtung schenkt. In diesem narrativen Zugang gilt das Grundparadigma, dass unsere menschlichen Identitäten immer in Erzählungen gefasst sind und dass «Geschichten-Erzählen» gar als conditio humana verstanden werden kann.

Jetzt kommen wir wieder auf die US-Wahlen zurück. Mit dem Schwerpunkt auf Geschichten, d.h. stories bzw. narratives, soll nicht gemeint sein, dass jeder einfach alles erzählen kann oder darf. Die Geschichten und Erzählungen, die unser Zusammenleben konstitu- ieren und um die es uns in der Narrativen Ethik primär geht, sind nicht Märchen oder fairy tales und schon gar nicht science fiction. Das Augenmerk auf solch imaginären Geschichten wäre ein ganz anderer Zugang (und hier kennt sich z.B. die Psychiatrie besser aus). In der Narrativen Ethik geht es um die ethische Analyse von realen Geschichten. Um «reale» Geschichten in einem ersten Schritt von "imaginären» Geschichten abzugrenzen, führt z.B. Hilde Lindemann eine hilfreiche vierstufige Charakterisierung ein. Reale Geschichten müssen den Zuhörenden eine (a) sinnvolle Bedeutung vermitteln, sie müssen (b) anschaulich und (c) selektiv sein und sie müssen (d) verbindende Elemente enthalten, die somit ein Gesamtverständnis verkörpern [1]. Fazit: Eine akkurate Geschichte muss also gewissen Gütekriterien genügen. Lindemann fügt noch drei weitere Punkte hinzu: Die Geschichte, die jemand erzählt, muss für Aussenstehende (e) nachvollziehbar sein. Sie muss sich (f) an den alltäglichen Handlungen des Betreffenden messen lassen. Und, sie muss diese Handlungen verständlich erklären und auch in ihren Gewichtungen bzw. Proportionen (g) stimmig wirken [2].

Natürlich, diese Analysekriterien werden niemand davon abhalten können, offensichtliche Lügen zu erzählen oder haarsträubende «alternative Fakten» zu verbreiten. Mir helfen die Kriterien von Lindemann trotzdem, und zwar beim Denken und beim Zuhören. Ich habe der Narrativen Ethik vor allem eine Erkenntnis zu verdanken. Geschichten müssen nicht notwendigerweise «wahr» sein. Aber damit ich selbst auf Geschichten meines Gegenübers stimmig reagieren kann, sollten sie mir zumindest «akkurat» erzählt sein. Wir alle sollten also an unseren eigenen Geschichten ernsthaft arbeiten, um sie «akkurater» zu gestalten, finde ich.

\section{Literatur}

1 Hilde Lindemann (2001): Damaged identities, narrative repair Ithaca, Cornell University Press.

2 Hilde Lindemann (2006): «Wrinkles in time: narrative approaches to ethics.» In: Zeithorizonte des Ethischen - Zur Bedeutung der Temporalität in der Fundamental- und Bioethik. Hrsg. von G. Pfleiderer und C. Rehmann-Sutter. Stuttgart: Kohlhammer, 123-32. 